\title{
Digitally switchable multi-focal element for wearable displays
}

\author{
Xuan Wang, Hong Hua
}

Xuan Wang, Hong Hua, "Digitally switchable multi-focal element for wearable displays," Proc. SPIE 11040, Optical Design Challenge 2019, 1104008 (27 February 2019); doi: 10.1117/12.2522820

SPIE Event: SPIE Optical Design Challenge, 2019, San Francisco, California, United States 


\title{
Digitally switchable multi-focal element for wearable displays
}

\author{
Xuan Wang*, Hong Hua \\ 3D Visualization and Imaging Systems Laboratory, College of Optical Sciences, University of \\ Arizona, 1630 East University Boulevard, Tucson, AZ 85721, USA
}

\begin{abstract}
An innovative concept is proposed for an optical element which offers the capability of rapidly switching the optical power of the system among multiple foci. The switchable multifocal element consists of a custom-designed freeform lens offering multiple discrete foci and a programmable high-speed liquid crystal shutter (LCS). The freeform lens is divided into patterned zones, through which multiple distinct foci are produced. The LCS consists of patterned zones corresponding to those zones of the freeform lens, which can be programmably switched on and off. By combining the multi-focal freeform lens and the LCS in a time-multiplexed fashion, a switchable multifocal element with high-speed, large aperture and large range of tunable power was achieved. The multifocal element also meets the other requirement of an ideal tunable optical element such as low-voltage control, robustness, and compactness. A proof-of-concept twofocal head mounted display was designed to demonstrate one application of the new switchable multifocal element. The design can provide a FOV of 40 degrees and angular resolution of 1 arc minutes in visual space in an $8 \mathrm{~mm}$ by $8 \mathrm{~mm}$ exit pupil.
\end{abstract}

Keywords: Multi-focal, switchable element, freeform optics

\section{INTRODUCTION}

A key limitation of the state-of-the-art VR/AR displays is the well-known vergence-accommodation conflict (VAC) problem due to the lack of the ability to create accurate focus cues ${ }^{1,2}$. One of the methods to address the VAC problem in conventional stereoscopic 3D displays is to rapidly render multiple image planes distributed at different focal distances to create true 3D light field displays, in which a key enabling technology is an optical element with electrically tunable optical power, known as vari-focal element (VFE). Many methods have been proposed to achieve a multi-focal plane display, such as deformable membrane mirror device (DMMD) ${ }^{3,4}$, liquid lens ${ }^{5,6}$, birefringence material ${ }^{7}$, liquid crystal lens ${ }^{8-10}$.

None of these existing tunable optics can fully satisfy all the requirements for an ideal tunable optical element for headmounted display which should have the properties of high-speed, large aperture, large range of tunable power, lowvoltage control, robustness, and compactness. For instance, the DMMD used in Hu's prototype is a defocus corrector from OKO technology which can offer an adequately high speed of over $1 \mathrm{KHz}$, but the range of tunable optical power is only about 1.3 diopters and the limited optical aperture is $10 \mathrm{~mm}$. Additionally, the driving voltage is over 200 volts, which is inappropriate for a wearable device. The liquid lens Liu used ${ }^{5}$ offers a large range of tunable optical power, but it has a limited response speed of $100 \mathrm{~Hz}$ and very small optical aperture of about $2.5-4 \mathrm{~mm}$. The electronically tunable lens offered by Optotune Inc. is another emerging technology based on the combination of optical fluids and elastic polymer membrane ${ }^{6,11}$. It affords a large range of tunable power, low voltage control, and a large optical aperture (up to $16 \mathrm{~mm}$ ). However, it has a response time of $2.5 \mathrm{~ms}$ and a settling time of $6-15 \mathrm{~ms}$, which makes the overall speed inadequate. Additionally, the optical power is sensitive to temperature and to gravity. The conventional liquid crystal lenses are subject to slow response speed. Recently, Lee et al. presented a hybrid solution of combining twisted nematic liquid crystal with conventional lenses ${ }^{10}$, and demonstrated the ability to achieve high-speed switching between two focal distances. To extend the number of foci, however, more sets of liquid crystal modules need to be stacked and will result in a large system volume.

In recent work ${ }^{12}$, we presented a novel design and prototype of a digitally switchable multi-focal lens (MFL) that offers the capability of rapidly switching the optical power of the system among multiple foci. The switchable MFL consists of a custom-designed freeform lens and a programmable high-speed liquid crystal shutter (LCS). Four foci were created by four concentric zones with different optical powers on one freeform surface. The focus switching is achieved by rapidly turning on the corresponding concentric apertures of the LCS to control the light transmission through different zones on the freeform lens. The main limitation of the proposed MFL is that segregating the freeform surface into spatially 
separated but concentric zones, each for a different focal depth, made it challenging to integrate such a device in a wearable display design. When implemented in a display system, each of its concentric focal zones of the MFL will be mapped to concentric regions at the eyebox. Unless the eyebox size is the same or smaller than that of the eye pupil, which would make the display challenging to use, the eye would need not be able to see the images rendered by all of focal zone at a single position simultaneously, which thus lead the device not ideal for vari-focal or multi-focal plane display architecture. .

In this paper, we propose a new design method to the freeform lens and the LCS that overcomes the key limitations of the previous design method and makes it feasible in a vari- or multi-focal head-mounted display. Instead of the concentric architecture, multiple distinct foci are produced through a set of specified patterned zones on the freeform lens. The LCS design modified to consist of patterned zones corresponding to those zones of the freeform lens. By combining the multi-focal freeform lens and the LCS in a time-multiplexing fashion, a multi-focal plane switchable lens can be achieved at a high switching speed (e.g. about $400 \mathrm{~Hz}$ ) or higher as we demonstrated previously ${ }^{12}$. Compared to other existing VFE technology, the proposed switchable MFL can afford all the desired properties such as high-speed, large aperture, large range of switchable power, low-voltage control, robustness, compactness, and scalability. A proofof-concept two-focal display was designed to demonstrate one application of the new switchable multifocal element. The design can provide a FOV of 40 degrees and angular resolution of 1 arc minutes in visual space in an $8 \mathrm{~mm}$ exit pupil. By scaling its size and choosing different zone distributions, we can have different focus configurations of the switchable MFL according to its application.

\section{BASIC CONCEPT}

The basic concept of the switchable multi-focal lens is illustrated in Fig. 1. It consists of a customized high speed LCS and a freeform lens. As shown in Fig. 1 b) and c), one surface of the freeform lens is divided into grid zones which are categorized into four sets (represented by the four colors in Fig. $1 \mathrm{c}$ )). The freeform surface is designed in a way that the optical power of each set is different, thus the whole freeform has a sequence of four foci. The LCS is a programmable device which can be rapidly switched on and off. The whole aperture of the LCS is also divided into similar specified sub aperture zones which are corresponding to the zone distribution of the freeform surface, shown as Fig. 1 a). By attaching the LCS to the freeform lens, the light transmitted through the freeform can be controlled by turning the LCS apertures on or off, therefore the focus of the lens can be switched among the four foci (e.g. F1, F2, F3, and F4 etc.).

a)

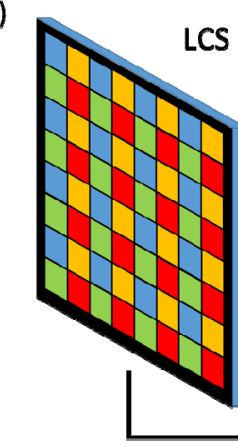

b)
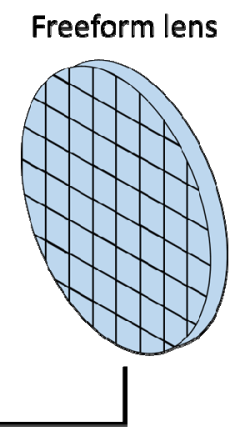

c)

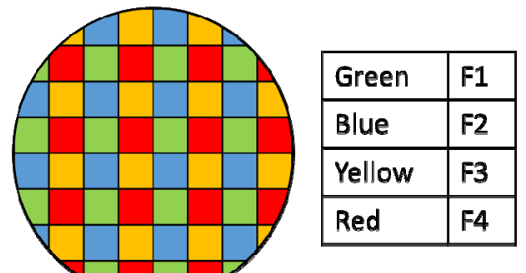

d)
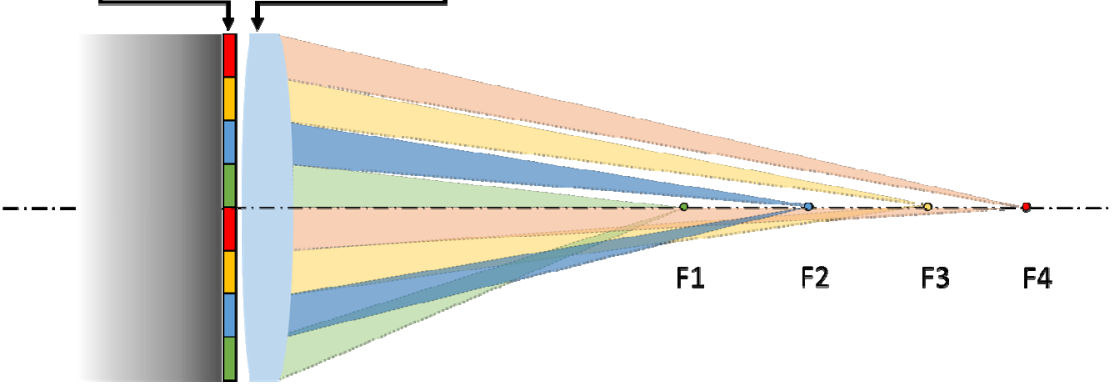

Figure 1. Basic concept of the switchable multi-focal element. a) LCS with specified grid patterns. b) Freeform lens divided into grid zones. c) Four grid zone sets on the freeform surface. d) Working principle of the switchable multi-focal element. 
The structure of the LCS is shown as Fig. 2. One layer of twisted nematic liquid crystal and two transparent electrode layers are sandwiched between the two glass substrates. The two electrode layers are also divided into four sets according to the aperture distribution of the LCS. Two orthogonal polarizers are separately attached to the outer surface of each glass substrate. All the zones of one aperture set connect to same electrode, thus each aperture set is controlled by one electrode. An aperture zone is set to be open with no voltage applied on its corresponding electrode, while it is set to be opaque with a $20 \mathrm{Vrms} 2 \mathrm{kHz}$ square wave applied. The detailed fabrication process and test result of the LCS have been discussed in our previous work ${ }^{12}$.

a)

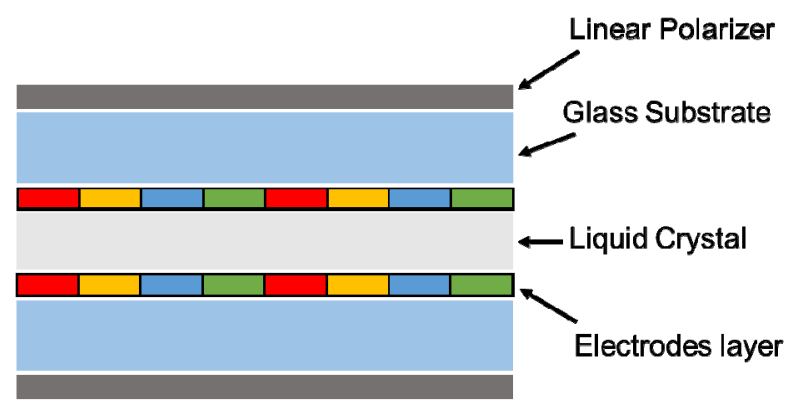

b)

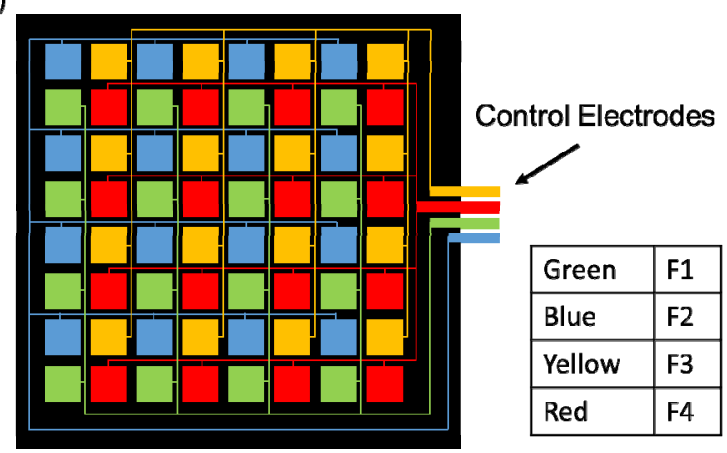

Figure 2. LCS Structure. a) Cross section of LCS. b) Aperture sets of the LCS and control electrodes.

Generally, when implemented in a display system, the vari- or multi-focal elements should be placed at the system stop to avoid deflecting system chief ray while the system focus changes, so the system maintains a constant angular resolution for different foci. In this configuration, the multi-focal lens is conjugate to the exit pupil also known as eyebox. Therefore, the exit pupil will be divided into sub viewing zones according to the aperture zone distribution of the LCS, shown as Fig. 3 a). By choose the size of the aperture zone on LCS and a reasonable pupil magnification, we can control the size of sub viewing zones. With appropriate size of these viewing zones, we can ensure that the observer can perceive the light from all the four foci no matter where the eye is at the eyebox, shown as Fig. 3 b). Obviously, smaller sub viewing zone size will make the light transmitted through each foci more evenly distributed. However, diffraction may become a big issue when the size of sub viewing zone is too small. Therefore, tradeoffs need to be made when determining the size of sub viewing zones.

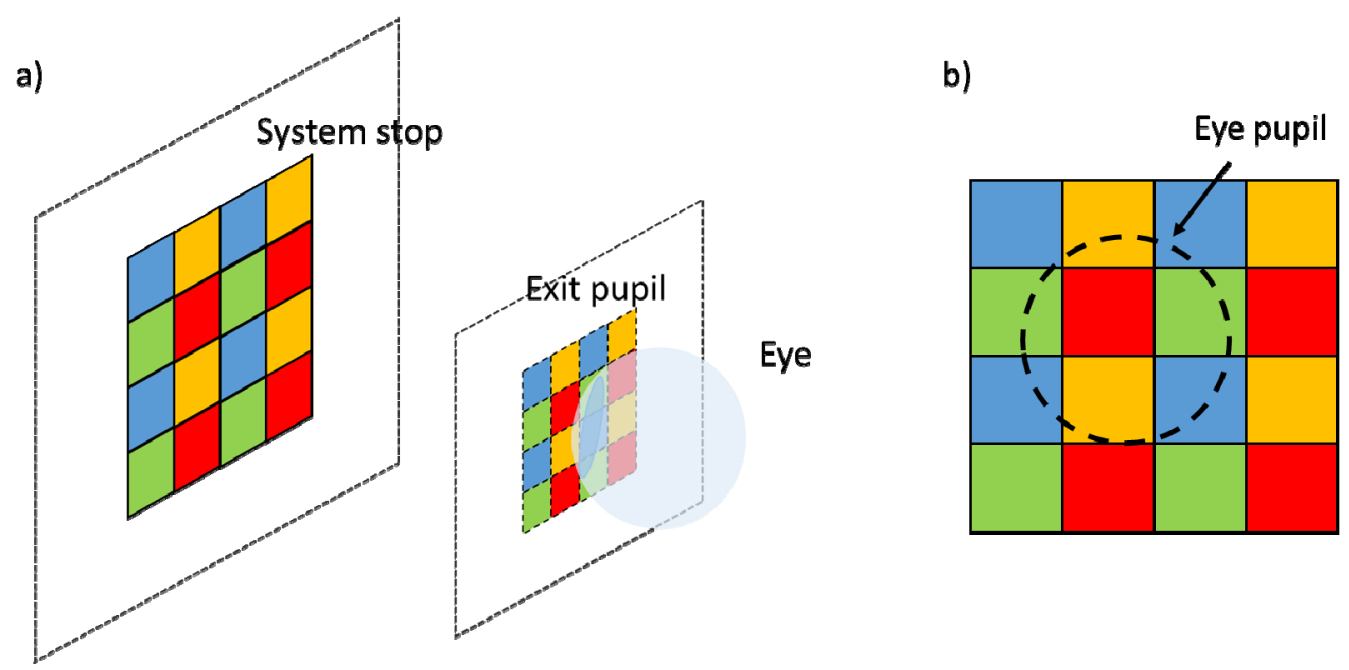

Figure 3. a) Sub viewing zones at exit pupil. b) Eye pupil at the exit pupil.

It's worth noting that although only 16 sub aperture zones are presented here, we can have more sub zones and the total aperture size of this multi-focal lens can be quite large. This will make the whole display system to have a sufficient eyebox size. Additionally, the focal length for each focus can also be customized to meet the system requirements. 


\section{PROOF OF CONCEPT SYSTEM DESIGN}

To demonstrate the concept, we designed a dual-focal display system illustrated as Fig. 4. A relay group, which consists of lens 1 and lens 2, makes the dual-focal lens conjugate to the exit pupil. The micro-display is placed at the focal plane of the short focus to make the corresponding virtual image present at infinity. The virtual image plane of the large focus is at 3 diopters. The system has a constant angular magnification so the two configurations share same chief ray shown as the purple ray in Fig.4. The red and brown rays represent part of the ray bundles for the two foci.

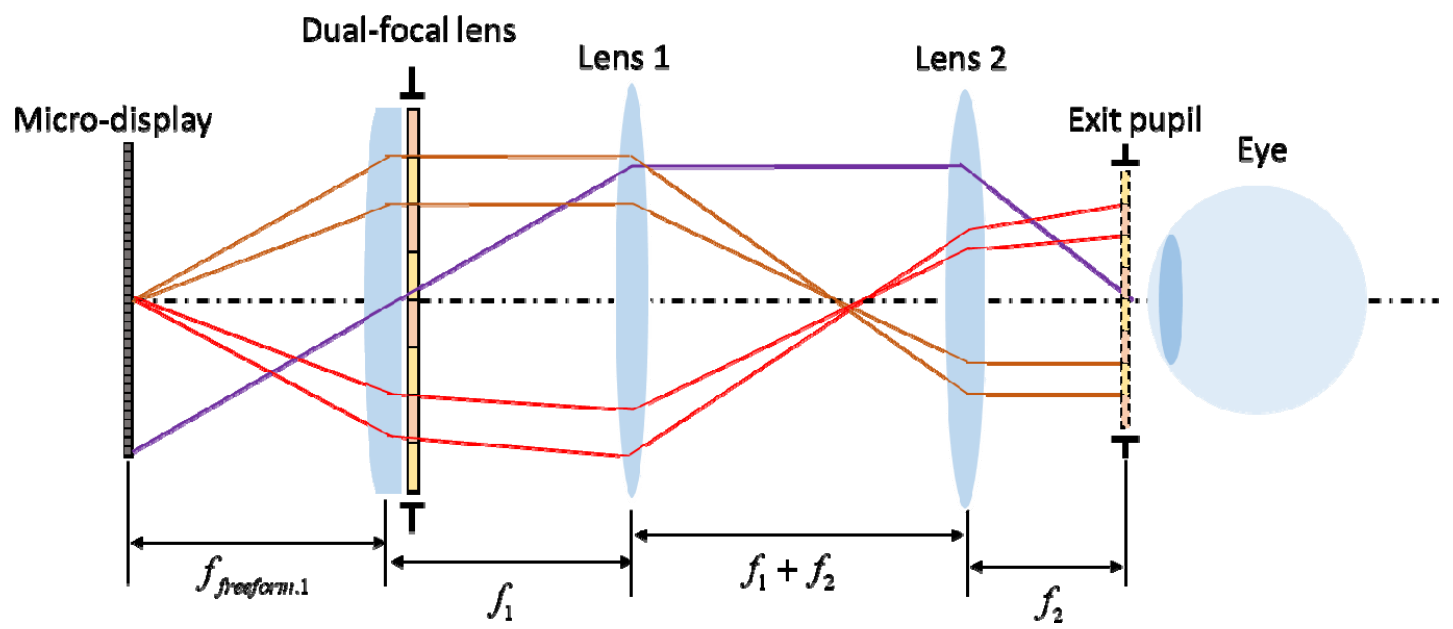

Figure 4. Scheme of a proof-of -concept dual-focal display system

Based on the optical scheme in Fig. 4, the key specifications of the designed prototype system are summarized in Table 1. The angular resolution is designed to fully match the fovea resolving ability of human eye which is around 1 arc minute. Considering a Sony organic light emitting display (OLED) offering an 8 - $\mu \mathrm{m}$ pixel pitch and a total of 1920x1080 color pixels, the full diagonal field of view (FOV) is about 40 degree. For a wearable device, the total length is limited to $150 \mathrm{~mm}$ and the eye relief is $20 \mathrm{~mm}$. An $8 \mathrm{~mm}$ exit pupil was created to allow eye movement of the observer when viewing the display. The size of sub viewing zone is $2 \mathrm{~mm}$ by $2 \mathrm{~mm}$, which is a balanced result of diffraction and foci distribution as we mentioned previously.

Table 1. Specifications of proof-of concept system.

\begin{tabular}{ll}
\hline Parameters & Value \\
\hline FOV & $40^{0}$ diagonal \\
Exit pupil diameter & $8 \mathrm{~mm}$ \\
Sub viewing zone size & $2 \mathrm{~mm} * 2 \mathrm{~mm}$ \\
Eye relief & $20 \mathrm{~mm}$ \\
Virtual Image position & Focus 1: Infinity \\
& Focus 2:3 diopters \\
Pixel size of Micro-display & $8 \mathrm{um}$ \\
Angular resolution & 1 arcmin \\
System total length & $150 \mathrm{~mm}$ \\
\hline
\end{tabular}




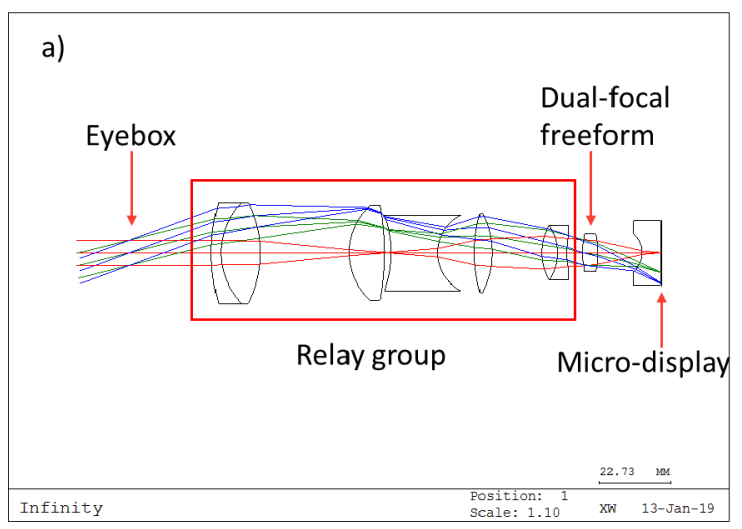

b)

b)

Figure 5. Layout of the designed display system. a) Infinity conjugate. b) 3 diopters conjugate.

Shown as Fig. 5 a) and b), we finally satisfied the requirements with a 7 piece system. Except the dual-focal freeform lens, all the lenses are conventional spherical lens and 2 doublets are used to correct the color aberration. The optical system design of the proposed system was very challenging due to not only the requirements for optimizing the optical performance at a high Nyquist frequency across a large FOV and a relative large exit pupil but also due to a strict control of the pupil aberration. Fig. 6. shows how well the pupil aberration is constrained. We can see that the footprint at the eyebox meet the way we designed. Fig. 7. plotted the MTF of the infinity conjugate and 3 diopters conjugate. The design yields an MTF above 0.1 at the spatial frequency of 62.5 cycles $/ \mathrm{mm}$ for all fields.
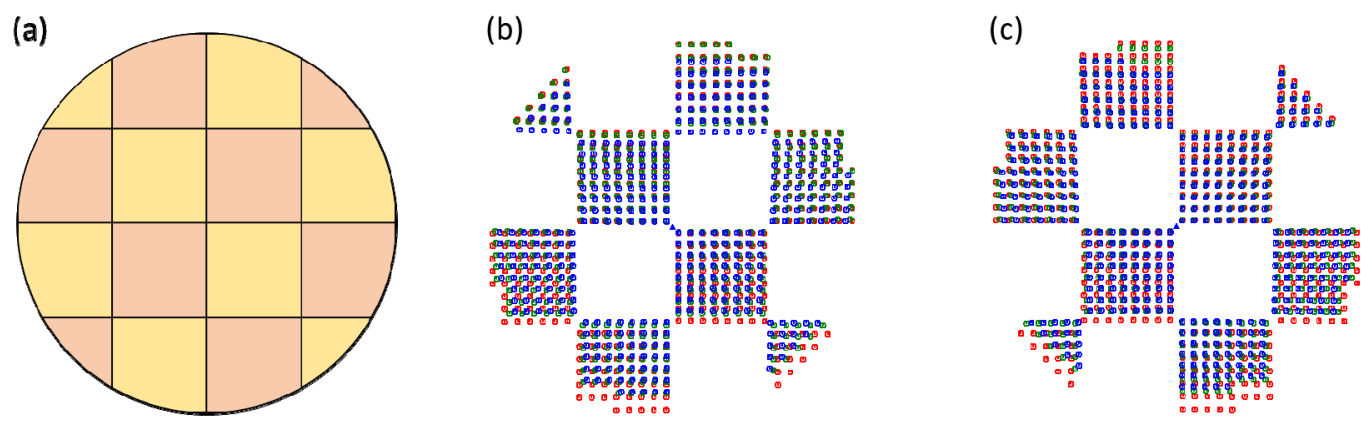

Figure 6. Footprint at the eyebox. (a) Schematic design. (b)Infinity conjugate and (c) 3 diopters conjugate for the system.
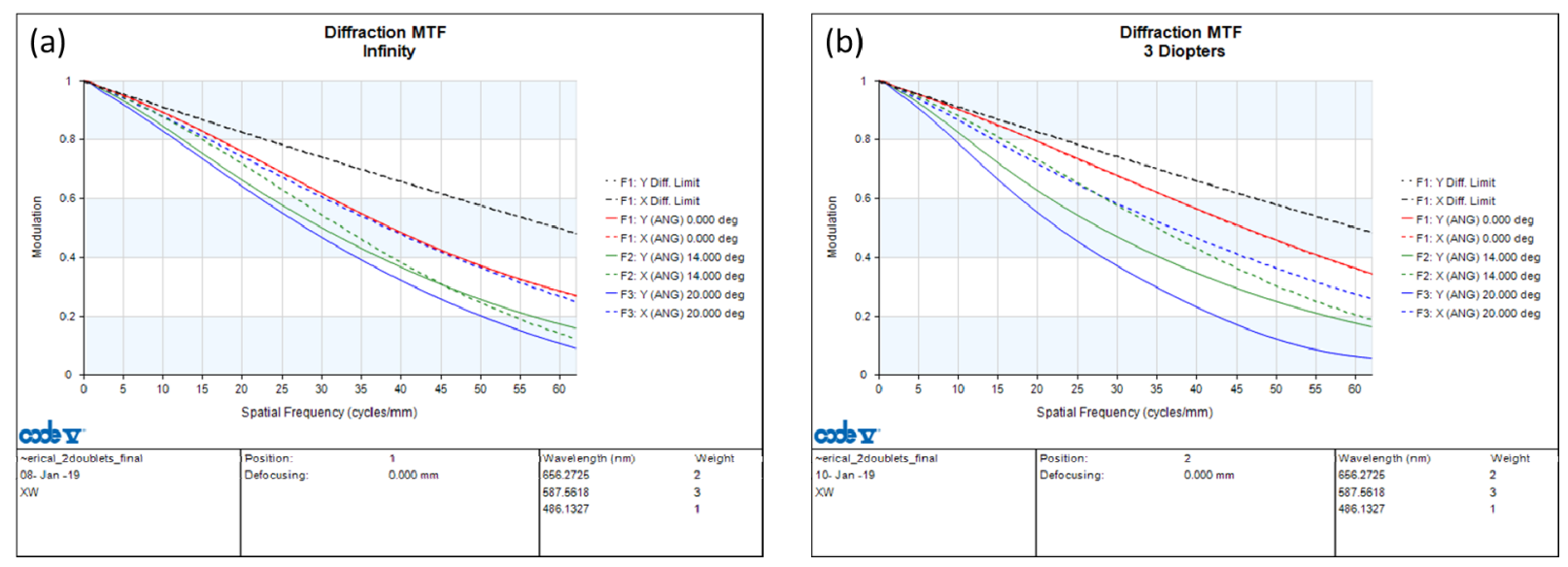

Figure 7. MTF plot of (a) Infinity conjugate and (b) 3 diopters conjugate of the proposed system. 
Figure 8 shows the simulated result of an UASF resolution target through the proposed system for the two foci, where Fig. 8(a) shows a sharp image of the UASF resolution target as the input and Fig. 8(b) and (c) shows the simulated 2D image of the target with its virtual image at infinity and 3 diopters, separately. We can see that the system has high image quality except for some distortion, which can be correct by prewraping the image displayed on the micro-display.
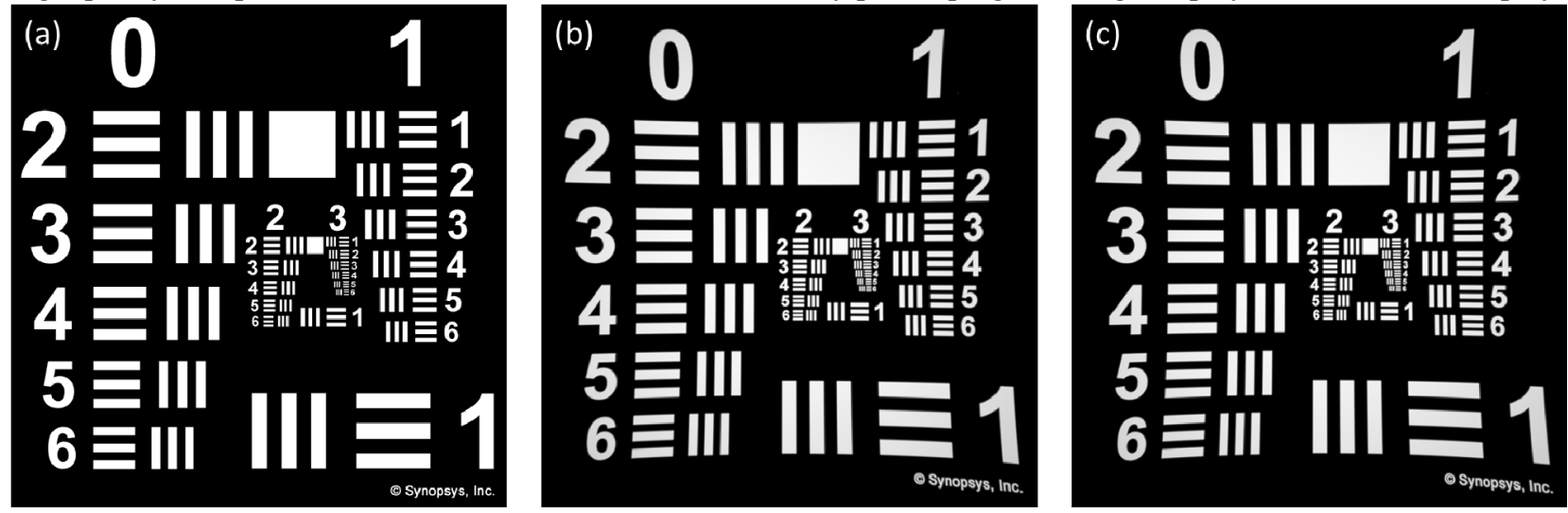

Figure 8. Image simulation. (a) Original image of an UASF resolution target. Simulated image of (b) Infinity conjugate and (c) 3 diopters conjugate of the proposed system.

\section{CONCLUSION}

In conclusion, we present a novel concept for a digitally switchable multi-focal element that offers the capability of rapidly switching the optical power of the system among multiple foci. The proposed multi-focal element can meet all the requirements of an ideal tunable optical element such as high-speed, large aperture, large range of tunable power, low-voltage control, robustness, and compactness. A proof-of-concept two-focal head mounted display was designed to demonstrate one application of the new switchable multifocal element. The design can provide a FOV of 40 degrees and angular resolution of 1 arc minutes in visual space in an $8 \mathrm{~mm}$ by $8 \mathrm{~mm}$ exit pupil.

\section{ACKNOWLEDGMENT}

Dr. Hong Hua has a disclosed financial interest in Magic Leap Inc. The terms of this arrangement have been properly disclosed to The University of Arizona and reviewed by the Institutional Review Committee in accordance with its conflict of interest policies.

\section{REFERENCES}

[1] J. Rolland, M. Krueger, and A. Goon, "Multifocal planes head-mounted displays," Appl. Opt. 39, 3209-3215 (2000).

[2] H. Hua, "Enabling focus cues in head-mounted displays," Proceedings of the IEEE, 105(5):805-24 (2017).

[3] B. T. Schowengerdt and E. J. Seibel, "True 3-D scanned voxel displays using single or multiple light sources." J. Soc. Inf. Disp.14, 135 (2006).

[4] X. Hu and H. Hua, "High-resolution optical see-through multi-focal-plane head-mounted display using freeform optics," Opt. Express 22(11), 13896-13903 (2014).

[5] S. Liu and H. Hua, "Time-multiplexed dual-focal plane head-mounted display with a liquid lens," Opt. Lett. 34, 1642-1644 (2009).

[6] H. Huang and H. Hua, "High-performance integral-imaging-based light field augmented reality display using freeform optics." Opt. Express 26(13), 17578-17590 (2018).

[7] G. D. Love, D. M. Hoffman, P. J. Hands, J. Gao, A. K. Kirby, and M. S. Banks, "High-speed switchable lens enables the development of a volumetric stereoscopic display," Opt. Express 17(18), 15716-15725 (2009). 
[8] H. Ren, D. W. Fox, B. Wu, and S. T. Wu, "Liquid crystal lens with large focal length tunability and low operating voltage," Opt. Express 15(18), 11328-11335 (2007) .

[9] A. Jamali, D. Bryant, Y. Zhang, A. Grunnet-Jepsen, A. Bhowmik, and P. J. Bos, "Design of a large aperture tunable refractive Fresnel liquid crystal lens," Appl. Opt. 57(7), 10-19 (2018).

[10] Y. H. Lee, F. Peng, and S. T. Wu, "Fast-response switchable lens for 3D and wearable displays," Opt. Express 24(2), 1668-1675 (2016).

[11] Optotune, Inc. "Fast electrically tunable lens EL-16-40-TC," http://www.optotune.com/products/focus-tunablelenses/electrical-lens-el-16-40-tc

[12] X. Wang, Y. Qin, H. Hua, Y. H. Lee, and S. T. Wu, "Digitally switchable multi-focal lens using freeform optics," Opt. Express 26(8), 11007-11017(2018). 